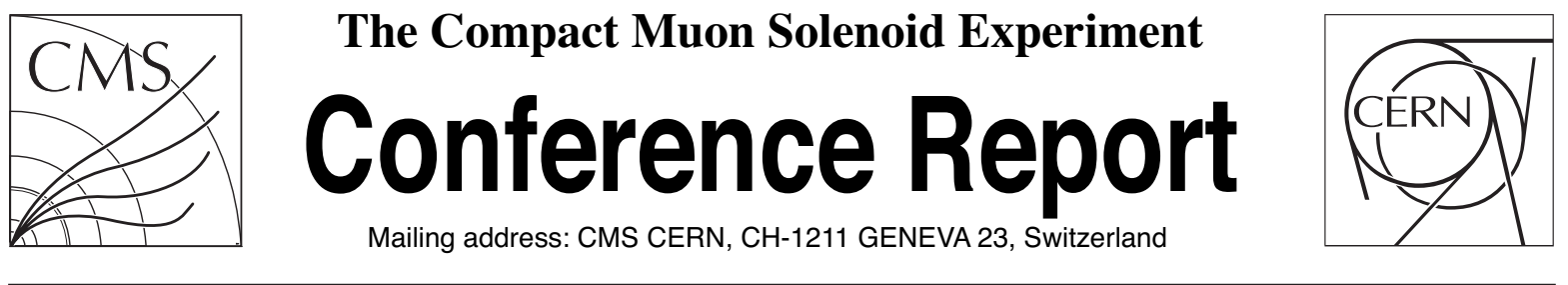

10 September 2008

\title{
Status of the CMS Silicon Strip Tracker and Commissioning Results
}

Ioana Anghel on behalf of the CMS Tracker Collaboration

\begin{abstract}
The main subsystems of the CMS Tracker have been assembled together in a dedicated large clean room at CERN, prior to the installation in CMS. Following the integration of the Tracker a long commissioning period took place, during which, up to $15 \%$ of the silicon modules were operated and read out simultaneously at different temperatures. Some of the achievements of this commissioning phase are discussed in details, like Tracker noise performance, signal-to-noise measurements and hit reconstruction efficiency. The Tracker was inserted in CMS in December 2007 and the commissioning of the full Tracker started. A sketch of the commissioning procedure followed at CMS and the actual status of the Tracker are presented.
\end{abstract}

Presented at PSD8: 8th International Conference on Position Sensitive Detectors, 1-5 September, 2008, Glasgow,United Kingdom 


\section{Introduction}

In order to study the physics of particles and forces at the smallest distances, accelerators are used to collide particles with great energy while detectors provide information on the characteristics of the collisions.

The Large Hadron Collider (LHC) is the world's largest and most powerful particle accelerator. One of the detectors built to study the LHC proton-proton collisions is the Compact Muon Solenoid (CMS) (Ref. [1]). This generalpurpose detector was designed for a wide range of physics goals, including: the discovery of Higgs boson, elucidation of the electroweak symmetry breaking mechanism, and the search for physics beyond the Standard Model. In order to archieve these goals, all detector subsystems are required to be at their best performance.

\section{CMS Tracker}

With about $200 \mathrm{~m}^{2}$ of active silicon area, $5.8 \mathrm{~m}$ long and a diameter of $2.5 \mathrm{~m}$, the CMS Tracker is the largest Silicon Strip Tracker ever built for high energy physics experiments. The Silicon Strip Tracker (SST) consists of four major subsystems, shown in Fig. 1: 16 half cylinder shells form the Inner Barrel (TIB) complemented by the Inner Disks (TID) - formed of 18 rings - cover the $20 \mathrm{~cm}<r<55 \mathrm{~cm}$ and $|z|<110 \mathrm{~cm}$ region, 688 rods which represent the Outer Barrel (TOB) covering aproximately $55 \mathrm{~cm}<r<120 \mathrm{~cm}$; and the same $\mathrm{z}$ range as the Inner part and $2 * 144$ petals representing the two End Caps (TEC), which complete the pseudorapidity coverage up to $|\eta| \sim 2.5$. The silicon detectors used at SST are made of one (thin - $320 \mu \mathrm{m}$ in the inner region corresponding to $r<600 \mathrm{~mm}$ ) or two (thick - $500 \mu \mathrm{m}$ in the outer region) daisy chained silicon sensors (Ref. [2]). In addition, some layers and innermost rings are equipped with special stereo modules, made of sandwiches of sensors with strips tilted by $100 \mathrm{mrad}$ with respect to each other, such providing also z information for barrel detectors and $\mathrm{r}$ information for disks.

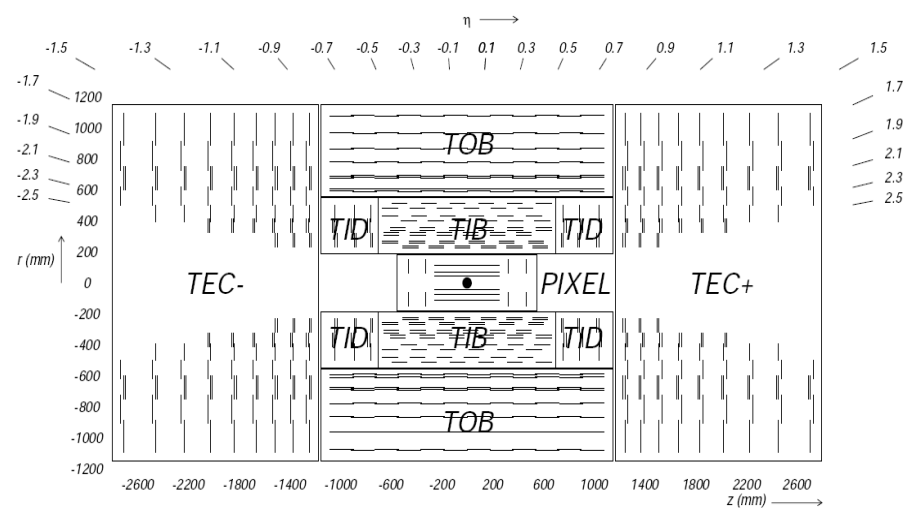

Fig. 1. CMS Tracker Layout
At design luminosity the detector will observe an event rate of aproximatively $10^{9}$ inelastic events each second. This leads to a number of formidable experimental challenges. The radiation hardness is absolutely necessary because of the high particle fluency. Is was proven necessary to cool up to $-20^{\circ} \mathrm{C}$ to reduce the defects in silicon due to the radiation. The density of the hits is very big and therefore a correct track reconstruction is almost impossible without a high granularity of the detector layers. The short time between bunch crossings, $25 \mathrm{~ns}$, has major implications for the design of the readout and trigger systems (Ref. [2]).

The quality of the construction and the performance of the components contained within the Tracker are of vital importance and must be thoroughly evaluated under realistic conditions before operation at LHC.

\section{Tracker Integration Facility (TIF)}

The SST construction proceeded during 2006 and 2007, tasks being distributed through much of the world. The final assembly of the Tracker was carried out in March 2007 in a large, purpose-built, clean area at CERN: the Tracker Integration Facility (TIF). This facility was instrumented with a significat fraction of the final infrastructure and services needed to operate, control and read out a sector of the Tracker that corresponds to $15 \%$ (on the $+Z$ side) of the entire detector.

The detector was commissioned and operated from March through July 2007 at the TIF, a period reffered as Sector Test, during which about 5 million Cosmic Ray Events were recorded. The slice of the Tracker to be operated was chosen in order to include portions of all subsystems, to have a good match with the segmentation of the cooling, control and readout systems, and to provide a good acceptance for cosmic muons. During the Sector Test, practical experience of the operation of the systems (Data Aquisition, Data Quality Monitoring, Control, Safety, Cooling etc.) was achieved.

The test progressed in an incremental way, beginning with testing parts of the sub-systems, then proceeding to a test of the barrel systems, and finally incorporating one endcap. The cooling plant used for the Sector Test was simpler than the final system and its cooling power was limited. A minimum operating temperature of $-15^{\circ} \mathrm{C}$ (Ref. [2]) was obtained, compared to $-25^{\circ} \mathrm{C}$ in the final system. The temperatures measured at the cooling tubes proved to be very stable with variations of less than $0.1^{\circ} \mathrm{C}$.

In order to understand the efficiency and alignment of the Tracker modules, a cosmic ray trigger was implemented in the TIF. The trigger design was constrained by space above and below the Tracker; in particular clearance below the Tracker allowed only $5 \mathrm{~cm}$ lead bricks for filtering low momentum tracks. Six scintillators were placed above the 
Tracker, in a fixed position; below the Tracker there was initially only one scintillator mounted on a movable suport structure; later a further set of four scintillators were added to increase the trigger acceptance. The trigger rates started from $3.5 \mathrm{~Hz}$ on the first configuration of the scintillators and got to $6.5 \mathrm{~Hz}$ in the last configuration. But since the Data Aquisition software (DAQ) was limited to about $3 \mathrm{~Hz}$ by the Front End Driver board (FED) readout, a trigger veto was implemented to keep the rate under that level.

Some of the achievements of this commissioning phase related to the Noise Performance, Hit Reconstruction and Signal Characterization are described below.

\subsection{Noise Performance}

The noise of a Tracker module is completely determined by the input capacitance at the APV25, which in turn is dominated by the silicon strips (128 strips/APV). Thus, one expects a linear dependence of the noise on the length of the silicon strip for all modules.

The pedestal and noise studies are part of the calibration of the detector. Pedestals have been measured before physics runs on daily basis. For a good reconstruction of the particle trajectory it is trivial to know the missing and noisy strips.

An important step in these analyses is applying the gain correction factor. This can be different for each pair of APVs connected to the same fiber. The height of the APV syncronization pulse (known as the tick mark height) depends on the operating voltage for each module, but it is also higly dependent on the fiber response. At TIF, in some studies it was used the tick mark height information to calculate the gain correction factor. There is a limitation though imposed by the different module operating voltages for different layers. An alternative way to the gain correction is the normalization of the noise for each fiber (256 strips) with respect to the mean of the noise from that fiber.

Faulty channels were studied both from the perspective of badly behaving modules or missing fibers and of individual bad channels. Dead fibers correspond to the broken fibers. Modules with known problems or which were badly behaving were removed either from DAQ or from the data analyses. The resulting fraction of missing modules was at the $0.5 \%$ level. The number of missing fibers in the Tracker was at the $0.1 \%$ level. The number of dead channels (see Table1) is very constant among several runs for all subdetectors, showing that the identification of these channels is clear and stable: the majority of the dead strips (70\%) were flagged in all runs (Ref. [2]). The noisy components is instead subject to fluctuations in particular for TOB and TID. On the other hand, only a small fraction of the noisy strips were noisy throughout the Sector Test.

Stability of noise performance was studied by taking pedestal and noise runs at different times and temperatures (from $+15^{\circ} \mathrm{C}$ to $-15^{\circ} \mathrm{C}$ ) when the Tracker was running in stable conditions. For constant coolant tempera- ture, the noise is stable to better than $0.5 \%$. Most importantly, the noise decreases with the decreasing temperature as expected from the laboratory studies made on the APV25 performance.

Fig. 2 shows the noise is proportional to the strip length, within the statistical uncertainty, as expected from the laboratory tests. The mean of the noise shows differences for the same strip length, but different module geometries. In the real data, effects such as temperature changes can also give rise to significant differences of the noise, even for sensors of the same length. The results are in good agreement with Monte Carlo predictions, except maybe for long sensors, but an exact parametrization of the noise is very hard to be done.

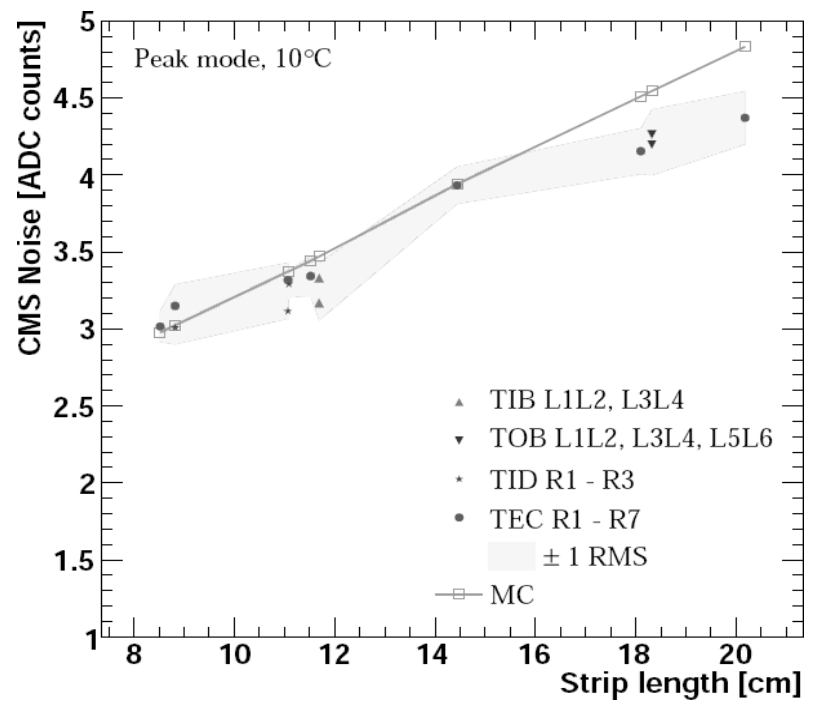

Fig. 2. Measured noise in the various sub-detectors as a function of strip length compared with the Monte Carlo parametrization. The band corresponds to the spread of the mean noise in the layer. (Ref. [6])

\subsection{Signal Characterization}

The signal performance of the Tracker is very important; it depends on several factors: charge collection on the silicon sensors, performance of the APV25 with well defined parameters, and performance of the full electronic chain. The energy $\left(S_{t o t}\right)$ (Ref. [2]) deposited in Tracker modules can be parameterized :

Table 1

The fraction of strips identified as bad during the Sector Test.

\begin{tabular}{|c|cc|}
\hline Detector type & $\begin{array}{c}\text { Dead Strips Noisy Strips } \\
(\%)\end{array}$ & $(\%)$ \\
\hline TIB & 0.05 & 0.04 \\
TID & 0.04 & 0.15 \\
TOB & 0.04 & 0.3 \\
TEC & 0.08 & 0.02 \\
\hline
\end{tabular}


$S_{t o t}=\frac{d E}{d x} t K$

where $K=\frac{1}{\cos \left(\theta_{3 D}\right)}, \theta_{3 D}$ is the angle of incidence of the track with respect to the silicon detector normal, and $t$ is the silicon thickness. The signal values are also normalized to the thickness of the silicon detectors, resulting $S_{r e n}$.

$S_{r e n}=\frac{S_{t o t}}{K}=\frac{d E}{d x} t$

This normalized Signal-to-Noise quantity $S_{r e n} / N$ is an ideal parameter for measuring the stability of the Tracker because it is largely independent of gain correction. It allows an acurate comparison of the performance of the modules in the same layer or at different temperatures or from run to run. The cluster noise $N$ is defined as $N=\sqrt{\sum_{i} \frac{N_{i}^{2}}{n_{\text {strips }}}}$ where $N_{i}$ is the noise of the i-th strip of the cluster and $n_{\text {strips }}$ is the number of strips of the cluster. $S_{\text {ren }} / N$ was calculated for all clusters from one layer. If the noise is constant for all strips in the cluster, then the cluster noise is independent of the cluster size and equal to the strip noise. All Tracker layers show a large $S_{r e n} / N$, in all cases higher than 26 .

$S_{\text {ren }} / N$ shows a very stable behavior for TIB and TOB with variations less than $0.3 \%$ when compared the runs taken at the same temperature. Some differences can be observed between the values for this ratio for the layers of the same detector. These may be due to the pitch density (mainly for TIB) and different geometries (TOB and TEC). The results for TOB are shown in Fig.3. $S_{r e n} / N$ increases with decreasing temperature, as expected from the noise temperature dependence. Similar results were obtained for TID and TEC, but the lower statistics per run gives rise to a higher statistical errors in the measurements.

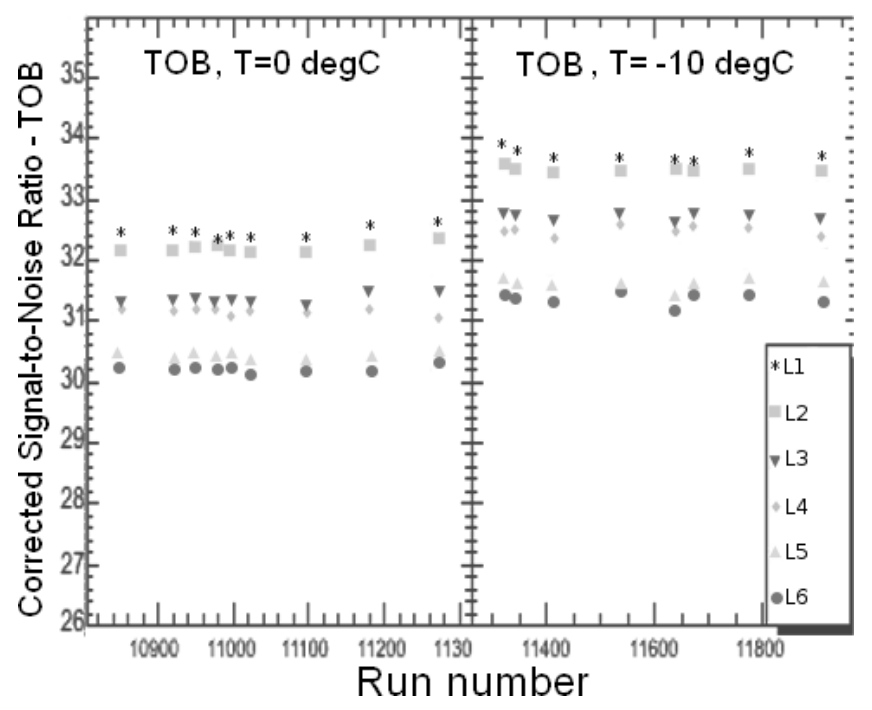

Fig. 3. The most probable values of the fitting funtion (landau) of $\mathrm{S} / \mathrm{N}$ corrected for the angle of the track vs the run number at different temperatures. The values for all TOB layers are shown. (Ref. [2])

\subsection{Hit Reconstruction Efficiency}

The efficiency of a Tracker module to observe a hit when tranversed by a particle, is one of the most important characteristics of the detector performance as this can be affected by losses in the silicon, electronic chain, or data aquisition and analysis. Efficiency has been measured by moving the track reconstruction algorithm to skip the layer under study during the pattern recognision phase. A sample of high quality events was selected by requiring only one track reconstructed by the CTF algorithm, one hit in the first TIB layer, one hit in the two outermost TOB layers, and at least four reconstructed hits(of which at least three matched from stereo layers). In addition an upper cut of $30^{\circ}$ for $\theta_{3 D}$ (see section 3.2) was applied to select tracks almost perpendicular to the modules.

In order to avoid genuine inefficiences present at the edge of the sensor and in the bonded region between two sensors additional cuts have been applied to restrict the region in which efficiency is measured. It resulted that the hit reconstruction efficiency exceeds $99.8 \%$ for all measured layers, as it is shown in Fig.4.

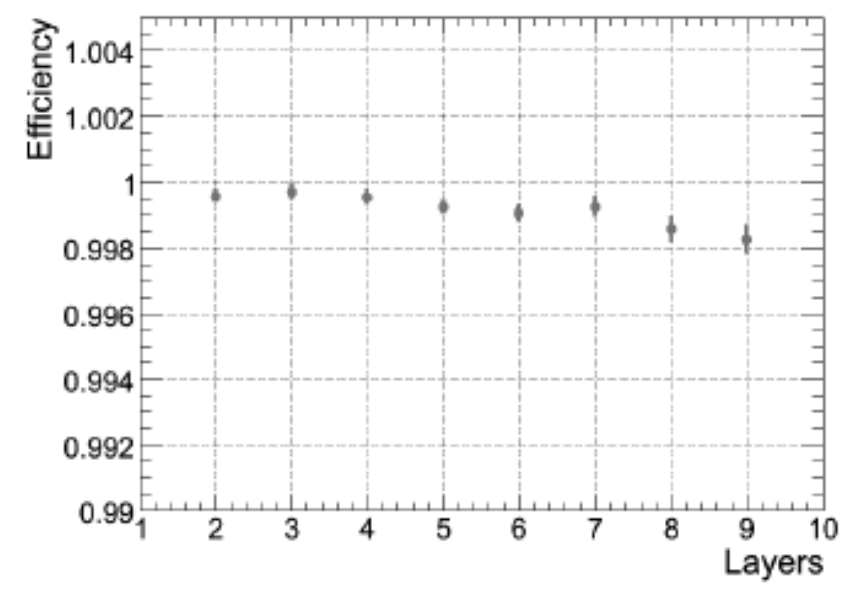

Fig. 4. Summary of hit reconstruction efficiency for all studied layers at $-10^{\circ} \mathrm{C}$. (Ref. [2])

\section{Commisioning Procedure}

The fully assembled Tracker was moved from TIF to CMS detector location (p5) in December 2007. The cables connection was completed in March 2008 and the first round of commissioning started immediately with a temporary cooling plant. After installing the final cooling plant, the second round of checkout and commissioning started in June.

The DAQ software developed to configure and manage readout of Tracker data is based on XDAQ applications (Ref. [5]), which also provided algorithms to commission the detector. There are a few steps which are followed during the commissioning. The Map between the Control system and Power Supply (PSU) and Readout systems is the first step that needs to be done in order to identify the con- 
nections. Once the connections are established, the channels readouts (amplitude of the signal - the tick mark) need to be synchronized. This is done by Internal Timing, which needs to accomodate for different fiber length, electrical data path.

After synchronization, a few actions are taken with the porpose of tuning the signal. For example, the tick mark itself needs to be tuned and this is the purpose of the Optical Gain Scan. During this step, the proper Analog Opto Hybrid $(\mathrm{AOH})$ settings are done: Laser bias, input signal scale factor. The Tuning of the APV pulse shape will assure: the homogeneity of the tracker response, the performances over time, the reducing of the systematics in $d E / d x$ studies (Ref. [3]). The Tracker is also synchronized with the LHC clock by running Latency Scan. The best Latency is set by reconstructing the pulse shape and then choosing the working point closest to the maximum.

The Pedestal and Noise runs are finalizing the Commissioning sequence. The information extracted during these steps is directly used in Physics Analyses.

The Commissioning procedures were validated and the quality of the SST is impressive: the vast majority of channels passed check out first time and the first look at performance looks good. Due to a huge effort, TIB, TID, TOB and TEC+ participated in CMS Global Run in early July 2008. Though the Tracker was not fully commisioned, the results obtained were really promising. Until the next CMS Global Run, in the middle of August 2008, the last part of the CMS Detector - the Pixel Detector - was inserted in CMS. Then, for the first time, the full CMS detector participated in a Global Run. At first look, the results are very good.

\section{Conclusions}

The Silicon Strip Tracker construction was finished in March 2007 at the TIF. During the Sector Test, SST has been operated in a stable and continuous manner for periods up to consecutive weeks. Noise and signal-to-noise results show a very stable behaviour of all subdetectors, and the values obtained are understood and are excellent. The signal-to-noise is larger than 26 for all layers/rings and the hit reconstruction efficiency is above $99.8 \%$. The level of bad strips is below $0.1 \%$. This commissioning actually demonstrated that the Tracker fully meets the experiment requirements and it has an excellent quality.

The Tracker commissioning at p5 is almost finished. In the middle of August 2008, the full CMS Detector, with the last piece (Pixel Detector) included, participated for the first time in a Global Run. At first look, the results are very good.

\section{References}

[1] M.Della Negra et al., "CMS Physics Technical Design Report: Volume 1, Detector Performance and Software", CERN LHCC-
2006-001, 2006

[2] L. Demaria et al., "Silicon Strip Tracker Detector Performance with Cosmic Ray Data at the Tracker Integration Facility ", CMS Note 2008 in preparation

[3] C.Delaere, L. Mirabito, "Timing of the CMS Tracker. Study of module properties.", CMS Note 2007/027 (2007)

[4] J. Cole, "Experience of Commissioning the CMS Silicon Strip Tracker ", Vertex08, 2008

[5] J. Gutleber, L. Orsini, "Software Architecture for Processing Cluster Based on I2O ", Cluster Computing, Volume 5 , 2002

[6] D. Giordano, "Calibration and Monitoring of the CMS Strip Tracker Detector “, Vertex08, 2008 